\title{
Impacto do traumatismo dentário na qualidade de vida de crianças e adolescentes: revisão crítica e instrumentos de medida
}

\author{
The impact of dental trauma on quality of life of children \\ and adolescents: a critical review and measurement instruments
}

Lívia Azeredo Alves Antunes ${ }^{1}$

Anna Thereza Leão ${ }^{2}$

Lucianne Cople Maia ${ }^{2}$

${ }^{1}$ Faculdade de Odontologia, Centro de Ciências Médicas, Universidade Federal Fluminense (Polo Universitário de Nova Friburgo). Rua Doutor Silvio Henrique Braune 22, Centro. 28625-650 Nova Friburgo RJ.

liviaazeredo@vm.uff.br

${ }^{2}$ Faculdade de Odontologia, Centro de Ciências da

Saúde, Universidade Federal do Rio de Janeiro.

\begin{abstract}
Dental trauma constitutes a public health problem with a marked prevalence among Brazilian children and adolescents. Furthermore, it can lead to irreparable dental loss, which makes it important to evaluate this condition using instruments of oral health related to quality of life (OHRQoL). Based on this, a review of the literature sought to expose indices that may assess the evaluation of quality of life among Brazilians with dental trauma, as well as discuss the state of the art of publications about this condition on their OHRQoL followed by a discussion of the output encountered on the subject. With this in mind the articles published from 1980 to June/2011 located on databases (Pubmed, VHL, Google Scholar) or manually in the references of selected publications were prioritized. It was revealed that there is no specific instrument for dental trauma. It is therefore necessary to assess the OHRQoL in children and adolescents with indices found in the literature. Once this has been done, as yet unresolved questions about the impact of dental trauma on OHRQoL can be answered.
\end{abstract}

Key words Children, Adolescents, Quality of life, Questionnaires, Dental trauma
Resumo O traumatismo dentário constitui-se em um problema de saúde pública com elevada prevalência, tanto em crianças como em adolescentes brasileiros. Aliado a isso, essa injúria pode ocasionar perdas dentais irreparáveis, o que torna importante avaliar o impacto dessa condição por meio de instrumentos de qualidade de vida relacionada à saúde bucal (QVRSB). Baseado nisso, a presente revisão de literatura, teve o propósito de expor indices existentes que possam avaliar a Qualidade de Vida $(Q V)$ em crianças e adolescentes brasileiros com traumatismo dentário, bem como apresentar o estado da arte dos trabalhos dessa condição na QVRSB dos mesmos e, em seguida, discutir a problematização sobre a produção encontrada a respeito do tema. Para tanto, priorizaram-se os artigos publicados de 1980 a junho/2011, pesquisados em bases de dados (Pubmed, BVS, Google Scholar) ou manualmente nas referências das publicações selecionadas. A partir deste trabalho, pôde ser constatado que não há instrumento específico para traumatismo dentário, sendo necessário utilizar indices voltados para avaliação da QVRSB em crianças e adolescentes presentes na literatura. Através deles, questões ainda não elucidadas sobre o impacto de traumatismos dentários na QVRSB poderão ser respondidas.

Palavras-chave Criança, Adolescente, Qualidade de vida, Questionários, Traumatismos dentários 


\section{Introdução}

Dentre os causadores de impacto negativo na qualidade de vida $(\mathrm{QV})$, o traumatismo dentário adquire um caráter especial por estar, juntamente com a doença cárie e o câncer bucal, entre os principais problemas de saúde pública em todo o mundo ${ }^{1}$.

O traumatismo dentário pode ocasionar perdas dentais irreparáveis em alguns casos, tanto no momento do acidente como do decorrer do tratamento ou até mesmo anos após. Dessa forma, esta condição pode criar sérios danos estéticos, psicológicos, sociais além de produzir significativos custos para a vítima do traumatismo ${ }^{2}$ No caso de crianças, esses danos afetam também seus pais e o profissional que presta o atendimento, uma vez que a sua resolução adequada e "definitiva" nem sempre é simples e rápida ${ }^{3}$.

A avaliação da qualidade de vida relacionada à saúde bucal (QVRSB) em crianças e adolescentes apresenta algumas particularidades ${ }^{4}$. No caso do indivíduo em desenvolvimento a peculiaridade mais importante é a diferença do grau de percepção que estes apresentam de si mesmos e do mundo, muito diferentes também quando comparado aos adultos ${ }^{5,6}$. Baseado nisso, nos últimos anos vêm se desenvolvendo instrumentos específicos para crianças e adolescentes que viabilizam a mensuração mais acurada do impacto dos problemas bucais sobre a sua QV.

Assim, para que a abordagem das crianças com traumatismos dentários seja feita de maneira efetiva é fundamental que as consequências desses problemas sejam avaliadas dentro de questionamentos referentes à sua repercussão sobre a $\mathrm{QV}$, uma vez que tratar unicamente sinais e sintomas de uma patologia não permite que o indivíduo usufrua de sua saúde integralmente. Tal avaliação acompanha um novo conceito de saúde que considera a qualidade de vida como bemestar com uma conotação multidimensional ${ }^{7}$.

Neste contexto, este trabalho tem por objetivo, por meio de uma revisão de literatura, expor índices existentes que possam avaliar a QV em crianças e adolescentes brasileiros com traumatismo dentário, bem como apresentar o estado da arte dos trabalhos dessa condição na QVRSB dos mesmos e, em seguida, discutir a problematização sobre a produção encontrada a respeito do tema.

\section{Aspectos metodológicos}

Para seguir um critério na realização dessa revisão narrativa da literatura priorizaram-se os artigos publicados de 1980 a junho de 2011 pesquisados eletrônicamente em bases de dados como Pubmed, BVS (Medline, Scielo, Lilacs, BBO) ou sites de busca como Google Scholar, a fim de avaliar resumos e títulos de estudos potencialmente relevantes. Nos casos em que o título e o resumo não fossem esclarecedores, o artigo foi acessado na íntegra. Os termos utilizados nas buscas bibliográficas eletrônica foram: criança, adolescente, qualidade de vida, questionários, traumatismos dentários. Após a seleção das publicações, suas referências também foram manualmente avaliadas a fim de encontrar artigos sobre o referido tema.

Como padrão de inclusão dos artigos buscou-se:

. Artigos de validação de instrumentos para avaliação da QVRSB para crianças e adolescentes de 0 a 14 anos (os originais e os de validação para língua portuguesa);

. Artigos que aplicavam intrumentos de QVR$\mathrm{SB}$ em crianças e adolescentes com traumatismo dentário.

Foram excluídas publicações regionais não indexadas, anais de congressos, teses, dissertações, livros e capítulos de livros.

Após a aplicação dos critérios de inclusão e exclusão foram selecionados 26 artigos. Os eleitos para utilização na presente revisão de literatura tiveram seus conteúdos analisados; os que versavam sobre validação de instrumentos de QVRSB, tanto os originais (6 artigos ${ }^{8-13}$ ) quanto os validados para o português (10 $\left.\operatorname{artigos}^{14-23}\right)$, foram obtidas suas respectivas indicações de aplicação; e os artigos sobre aplicação de intrumentos de QVRSB em crianças e adolescentes com traumatismo dentário (10 $\operatorname{artigos}^{2,24-32}$ ) foram analisados sobre a população em que foram empregados, as suas metodologias e os resultados obtidos.

\section{Revisão da literatura}

Instrumentos de medida para avaliação do impacto da QVRSB de crianças e adolescentes

O primeiro trabalho descrevendo um questionário construído com o objetivo de mensurar a QVRSB de crianças e adolescentes foi 
publicado somente em 2002, o COHQOL ${ }^{\circledast}$ (Child Oral Health Quality of Life Instrument $)^{8}$, um conjunto de instrumentos de escalas multidimensionais, desenvolvido em inglês, no Canadá, que mede os efeitos negativos das alterações bucais de crianças de 6 a 14 anos, e também, a percepção dos pais e o impacto sobre a vida familiar. Devido à grande variabilidade na capacidade cognitiva da criança de acordo com a idade, esse conjunto instrumentos foi dividido em cinco questionários $^{8-10}$ : o Parental-Caregiver Perceptions Questionnaire (P-CPQ) - também conhecido como Parental Perceptions Questonnaire (PPQ) - que avalia o impacto nas crianças sob a percepção dos pais e cuidadores; o Family Impact Scale (FIS) que avalia o impacto sobre a família; e o Child Perceptions Questionnaire (CPQ) que avalia o impacto da percepção das crianças por instrumentos segundo faixas etárias: 6-7 (CPQ $\left.{ }_{6-7}\right), 8-10\left(\mathrm{CPQ}_{8-10}\right)$ e 11-14 $\left(\mathrm{CPQ}_{11-14}\right)$ anos.

A partir de então, baseado no modelo conceitual proposto por Locker ${ }^{33}$, uma série de questionários são relatados na literatura odontológi- ca avaliando a QV das crianças e adolescentes como: o Child Oral Impacts on Daily Performances (Child-OIDP) ${ }^{11}$ que relaciona a influência sobre a qualidade de vida de crianças/adolescentes de 11 a 12 anos, avaliando oito atividades diárias; o Early Childhood Health Impact Scale (ECOHIS $)^{12}$ que é um instrumento para pré-escolares ( 3 a 5 anos) e seus familiares; e o Child Oral Health Impact Profile (COHIP) $)^{13}$ um instrumento elaborado para ser respondido pelos pais e pelas crianças visando avaliar tanto aspectos positivos quanto negativos da QVRSB.

Dentre esses índices previamente citados, quais poderiam ser aplicados a crianças e adolescentes brasileiros com traumatismos dentários? O ideal é estar lançando mão de instrumentos presentes na literatura que já estão validadas na língua portuguesa, conforme sintetizados no Quadro 1. Dos índices mencionados anteriormente, apenas o $\mathrm{CPQ}_{6-7}$ e o COHIP não apresentam-se validados.

Quadro 1. Resumo das versões validadas para o português dos instrumentos de QVRSB para crianças e adolescentes.

\begin{tabular}{|c|c|c|c|c|c|}
\hline Instrumento & $\begin{array}{l}\text { Faixa } \\
\text { etária }\end{array}$ & Respondente & Domínios & $\begin{array}{l}\text { No de } \\
\text { itens }\end{array}$ & $\begin{array}{l}\text { Referência } \\
\text { Versão em } \\
\text { Português }\end{array}$ \\
\hline \multirow[t]{2}{*}{$\mathrm{CPQ}^{*}{ }_{11-14}$} & 11-14 anos & Criança & \multirow{4}{*}{$\begin{array}{l}\text { Sintomas bucais, } \\
\text { Limitações funcionais, } \\
\text { Bem-estar emocional, } \\
\text { Bem-estar social }\end{array}$} & 37 & $\begin{array}{l}\text { Goursand et al. }{ }^{14} \\
\text { Barbosa et al. }{ }^{15}\end{array}$ \\
\hline & & & & 8 e 16 & Torres et al. ${ }^{16}$ \\
\hline $\mathrm{CPQ}_{8-10}^{*}$ & $8-10$ anos & Criança & & 25 & Martins et al. ${ }^{17}$ \\
\hline $\mathrm{P}^{-\mathrm{CPQ}^{*}}$ & 6-14 anos & $\begin{array}{c}\text { Pais/ } \\
\text { Responsáveis }\end{array}$ & & 31 & $\begin{array}{l}\text { Gousand et al. }{ }^{18} \\
\text { Barbosa et al. }{ }^{19}\end{array}$ \\
\hline CHILD-OIDP & 11-12 anos & Criança & $\begin{array}{l}\text { Atividades diárias relacionadas } \\
\text { ao desempenho físico, } \\
\text { psicológico e social }\end{array}$ & 8 & Castro et al. ${ }^{20}$ \\
\hline ECOHIS & 3-5 anos & $\begin{array}{l}\text { Pais/ } \\
\text { responsáveis }\end{array}$ & $\begin{array}{l}\text { Subescala criança } \\
\text { Subescala família }\end{array}$ & 13 & Scarpelli ${ }^{21}$ \\
\hline $\mathrm{FIS}^{* \S}$ & 6-14 anos & $\begin{array}{c}\text { Pais e } \\
\text { familiares }\end{array}$ & $\begin{array}{c}\text { Atividade dos pais/família } \\
\text { Emoções dos pais } \\
\text { Conflito familiar } \\
\text { Encargos finaceiros }\end{array}$ & 14 & $\begin{array}{l}\text { Barbosa et al. }{ }^{22} \\
\text { Goursand et al. }{ }^{23}\end{array}$ \\
\hline
\end{tabular}

* instrumentos integrantes do COHQOL ${ }^{\circledR} ;{ }^{* \S}$ instrumentos para avaliação do impacto da condição bucal da criança e adolescente na vida familiar 


\section{Aplicação do instrumento de QVRSB em crianças e adolescentes com traumatismo dentário}

A primeira vez que o termo qualidade de vida é relacionado em periódicos de medicina foi na década de 60, e, nos periódicos de odontologia, na década de $90^{34}$. Os traumatismos dentários foram primeiramente avaliados como causadores de impacto na QVRSB, em 2002, no trabalho de Cortes et al. ${ }^{2}$ realizado em crianças brasileiras referente a dentes permanentes. Neste estudo, os autores relatam que, até então, nenhum levantamento epidemiológico foi feito sobre o impacto sociodental de dentes anteriores traumatizados e pouco era conhecido sobre como as crianças sentem-se, sobre como é ter dentes incisivos fraturados e os possíveis impactos psicossociais e emocionais no seu comportamento. Assim, por meio de um estudo caso-controle (2:1), perfazendo uma amostra de 189 crianças (63 casos e 126 controles), o objetivo de Cortes et al. ${ }^{2}$ foi obter informações sobre os impactos sociodentais de escolares brasileiros de 12-14 anos, com dentes anteriores fraturados e não tratados. Como instrumento de medida foi utilizado o Oral Impact Daily Performance (OIDP). Na época ainda não havia a versão voltada para a faixa etária (Child-OIDP) nem a versão validada em português. Eles observaram que crianças com fraturas estão mais propensas a relatar impacto para comer, limpar os dentes, sorrir, ficam mais envergonhadas, demonstram maior irritação e não gostam de encontrar pessoas. Consequentemente, crianças com fratura demonstram ser mais significantemente insatisfeitas com a aparência do que as sem injúria² .

O trabalho seguinte relacionado à avaliação do traumatismo dento-alveolar a QVRSB, também foi realizado no Brasil, em 2007, com adolescentes entre 10 e $19 \operatorname{anos}^{24}$. Este estudo tipo caso-controle (1:4), em que os casos avaliados eram de pacientes traumatizados tratados e o controle foi feito com paciente sem trauma, investigou a magnitude do impacto do tratamento de fraturas coronárias na qualidade de vida utilizando, como Cortes et al. ${ }^{2}$, o OIDP. A hipótese de que adolescentes sofrendo de fratura de esmalte e dentina apresenta maior prevalência de impacto dental na vida diária, mesmo tendo recebido tratamento quando comparado a outros sem história de trauma, foi comprovada. Isso revela que apesar da restauração, a ocorrência das consequências do trauma não pode ser eliminadas, mas minimizadas.
Na mesma linha de avaliação de impacto do traumatismo na QVRSB, Fakhuddin et al. ${ }^{25}$, em Ontario, delineou um estudo para avaliar o impacto social na QV causado por traumatismo dentário. Especificamente, o objetivo dos autores foi avaliar a QV de pessoas tratadas e não tratadas realizando um estudo do tipo caso-controle, como os dos autores anteriormente citados, Cortes et al. ${ }^{2}$ e Ramos-Jorge et al. ${ }^{24}$. Neste trabalho, a amostra constituiu-se de 270 adolescentes de 12 a 14 anos que relataram 1 ou 2 traumas. $\mathrm{O}$ instrumento utilizado foi o $\mathrm{CPQ}_{11-14}$. Observaram que crianças com trauma dental de incisivos superiores não tratadas tinham mais dificuldade na mastigação e evitavam sorrir. Nessa amostra, o impacto na subescala bem-estar social foi maior do que o impacto nas funcional e bem-estar emocional. Aquelas crianças com injúrias dentais não tratadas experimentavam maior risco de impacto social negativo, limitação física e funcional na sua rotina diária do que aqueles sem injúria, concordando com o estudo de Cortes et al. ${ }^{2}$ de que o traumatismo dental gera impacto na qualidade de vida de adolescentes.

Locker $^{26,27}$ em 2007 e 2008 utiliza uma mesma amostra de crianças canadenses de 11 a 14 anos que tiveram experiência de trauma e seus respectivos pais para, no primeiro trabalho, acessar as diferenças socioeconômicas na QVRSB e, no segundo trabalho, avaliar a validade da associação de uma única pergunta de saúde bucal feita aos pais com os itens do $\mathrm{CPQ}_{11-14}$ (versão curta). Não foram trabalhos específicos para analisar o impacto de traumatismo dental na QV, pois foram incluídas outras condições. Apesar do impacto do traumatismo não ser o foco da pesquisa, no estudo de 2007, tem-se acesso aos números do impacto do traumatismo classificado de acordo com o Índice de traumatismo dentário (Dental Trauma Index - DTI). A média do escore $\mathrm{CPQ}_{11}$ ${ }_{14}$ (versão curta) variou de 10 a 32 com média de 12,9 e variação de 4,2. Classificando de acordo com o DTI, tanto no código de 1-5 quanto no de 2-5, o maior impacto foi gerado nos adolescentes que tinham 2 ou mais dentes afetados tendo o segundo, o de acometimentos mais severos, obtido as maiores médias (13,7 versus 16,4).

Com objetivo de comparar formas específicas e genéricas do índice Child-OIDP, dentre outros grupos analisados, uma amostra de pacientes de 11 a 12 anos que tinham necessidades normativas por terem sofrido traumatismo dental foram incluídas no estudo de Bernabé et al. ${ }^{28}$. Esse trabalho é interessante, pois inicia uma busca por respostas para a avaliação de instrumentos mais 
específicos para cada situação que possa gerar impacto na QV. Neste trabalho, observou-se que quando utilizado o instrumento especifico detectou-se discriminação entre os grupos com e sem necessidade normativa para trauma.

Outro estudo, de Berger et al. ${ }^{29}$, propôs quantificar a percepção de dor produzida pela injúria dental e seu tratamento utilizando escala visual analógica testada em pacientes e pais, bem como avaliar efeitos de injúria dental severa na QVRSB de crianças e adolescentes e suas famílias usando questionários do COHQOL ${ }^{\oplus}$. Quanto ao aspecto de avaliação da QVRSB, os autores analisaram a influencia do traumatismo dento-alveolar em 23 crianças de 8 a 14 anos e seus respectivos pais. A percepção das crianças foi obtida de acordo com a faixa etária, 8-10 e 11-14 anos, respectivamente com os instrumentos $\mathrm{CPQ}_{8-10}$ e $\mathrm{CPQ}_{11-14}$. A percepção dos pais quanto ao impacto na criança foi através do PPQ e o impacto na família pelo FIS. Os questionários foram aplicados no baseline (antes de 1 mês) após 6 meses, e após 12 meses. O COHQOL ${ }^{\oplus}$ demonstrou efeito profundo e continuado nas crianças e seus pais após injúrias dentais severas. Aos 6 meses, a média do CPQ aos 8-10 anos e 11-14 diminuiu. Isso demonstra a adaptação das crianças sem tratamento ao seu estado pós-injúria. As mensurações dos efeitos na condição da criança na família, obtida pelo FIS, não apresentou diferença estatística entre os momentos. O PPQ foi maior no início do que aos 12 meses tanto nas crianças com 8-10 anos quanto nas de 11-14 anos e redução após os 12 meses. Ao final de 1 ano, crianças ainda tinham os 4 domínios avaliados (sintomas orais, funcionais, bem-estar emocional e social) afetados, enquanto os pais relatavam efeitos limitados aos sintomas orais e limitações funcionais. Essa diferença nos relatos de sintomas suporta o conceito de que pais devem servir apenas como complementar na avaliação da $\mathrm{QV}_{29}$.

O trabalho de Bendo et al. ${ }^{30}$, constitui o primeiro na população brasileira de crianças a investigar o impacto de injúrias dentais traumáticas na QVRSB com instrumento específico, uma vez que estudos citados anteriormente ${ }^{2,24}$, nessa população, aplicaram o OIDP. Este estudo transversal foi realizado a partir de uma amostra representativa de 1612 crianças na faixa etária de 11 a 14 anos atendida em escolas pública e privada da cidade de Belo Horizonte, Brasil. Para tal avaliação foi utilizado o instrumento $\mathrm{CPQ}_{11-14}$ autoadministrado. Primeiramente foram detectadas as crianças diagnosticadas com traumatismo dentário não tratado $(n=219)$ e tratado $(n=64)$. A comparação entre esses grupos constatou que não há associação do traumatismo dental com sintomas orais, limitações funcionais ou bem estar emocional. No entanto, as crianças com traumatismo dental nos dentes anteriores experimentaram impacto social negativo, principalmente em relação a sorrir ou a dar risadas e a estar preocupado sobre o que as outras pessoas pensam ou poderiam falar.

De acordo com Abanto et al. ${ }^{31}$ fatores socioeconômicos poderiam confundir a associação de acometimentos bucais com QV, assim, os autores avaliaram a relação entre fatores socioeconômicos com o impacto da cárie da primeira infância (ECC), lesões traumáticas dentárias e maloclusão na QVRSB por meio da aplicação do ECOHIS a 260 pais de crianças de 2 a 5 anos. Como conclusão, observaram que dentre os fatores causadores de impacto na QV, a gravidade da ECC e uma renda familiar mais baixa teve um impacto negativo sobre a QVRSB de pré-escolares e seus pais.

Porrit et al. ${ }^{32}$, realizaram estudo para avaliar potenciais fatores influenciadores do traumatismo dentário na QV. Para realização desse estudo utilizaram uma amostra de 244 crianças atendidas em hospital dentário do Reino Unido. Os fatores avaliados, variáveis clínicas (número de dentes feridos; gravidade da lesão dental; visibilidade da lesão, tempo desde a lesão, e número de consultas dentárias atendidas), demográficas e psicossociais (relação da QVRSB e qualidade de vida geral), foram coletados no início do estudo, e as variáveis de desfecho foram analisadas novamente em seis meses de seguimento. $\mathrm{O}$ resultado identificou que as meninas tinham mais probabilidade de relatar maior nível de impacto tanto na QV geral quanto na QVRSB que os rapazes após uma lesão traumática nos incisivos permanentes. Além disso, foi observado que variáveis clínicas não foram preditores significativos na QV da criança após o trauma dento-alveolar no início ou após 6 meses de acompanhamento.

\section{Problematização sobre a produção encontrada a respeito do tema}

Na consulta à literatura foi observado que um maior conhecimento sobre o impacto de traumatismos dentários na QV de crianças em diversas faixas etárias, cujo comportamento cognitivo difere, é de suma importância para o desenvolvimento de medidas que visem, não só prevenir sua ocorrência e a forma de abordá-los, mas também enfatizar os cuidados com as repercussões sociais e psicológicas causadas nos pais, familia- 
res e na própria criança. Esse fato denota a extrema importância da utilização de instrumentos de QV validados para a população pediátrica.

O emprego de instrumentos de QVRSB para mensurar o impacto causado pelo traumatismo dentário na QV deve ser utilizado, uma vez que a prevalência dessa condição em crianças brasileiras é alta variando de $9,7 \%$ a $39,1 \%$ para a dentição decídua ${ }^{35-39}$ e de $10,5 \%$ a $17,3 \%$ para a permanente ${ }^{40,41}$. No entanto, uma vez que não há um instrumento de QV específico para esse fim, a literatura contempla diversos outros para a avaliação do impacto da condição bucal na QV da criança e do adolescente, como também na vida familiar, validados na língua portuguesa.

De acordo com Andeassen ${ }^{42}$, o traumatismo dental apresenta grande importância epidemiológica no contexto da Saúde Pública, uma vez que é muito frequente na infância e na adolescência, ocorrendo em 2 a cada 3 crianças antes da idade adulta. No entanto, sua taxa de publicação é inferior a $1 \%$ - completamente fora de proporção ao tamanho do problema ${ }^{42}$. No que concerne às publicações que avaliam o impacto do traumatismo dentário na QVRSB de pacientes infantis esse número é ainda muito menor.

Pode-se observar que na maior parte das pesquisas sobre o impacto do traumatismo dentário na QVRSB as avaliações foram em pacientes que haviam sofrido traumatismo dentário comparando-os a grupos que receberam ou não tratamento. Também se pôde observar que as fraturas foram os tipos de traumas mais investigados. $\mathrm{Na}$ realidade, seria de grande interesse para o meio científico avaliações na QVRSB que contemplassem também os traumatismos no tecido de suporte, principalmente para as crianças, uma vez que, de acordo com Jesus et al. ${ }^{43}$, esse tipo de injúria traumática é o mais frequente na dentição decídua, tendo também o agravante de poder gerar diversas sequelas na dentição permanente.

Abanto et al..$^{31}$ relacionando fatores socioeconômicos ao traumatismo dentário e Porrit et al. ${ }^{32}$ investigando como fatores clínicos e demográficos podem influenciar no impacto dos traumatismos dentários em crianças começaram a abrir novas perspectivas de investigação, no entanto, muitas questões referentes ao impacto que o traumatismo dentário pode gerar ainda estão pouco elucidadas: Como seria o impacto em diversas populações? Haveria diferença do impacto no momento imediato do traumatismo dentário? Como seria o impacto de acordo com os tipos de injúria traumática? Será que o impacto seria diferente de acordo com a faixa etária? Como ficaria o impacto da criança e do adolescente após o tratamento? Haveria variação? Será que os instrumentos seriam sensíveis à variação?

\section{Considerações finais}

A partir deste trabalho, pôde ser constatado que dentre os diversos instrumentos encontrados na literatura voltados para a avaliação da QVRSB em crianças e adolescentes não há um específico para a relação traumatismo dentário e QV. Sendo assim, é necessário lançar mão de índices presentes na literatura e, no caso de se realizar essa investigação na população brasileira, seria interessante a utilização de instrumentos validados na língua portuguesa. Além disso, são observados poucos estudos sobre o tema e muitas questões sobre avaliação do impacto do traumatismo dentário sobre a QVRSB ainda poderão ser respondidas em estudos futuros. Dessa forma, pode-se constatar que o campo de avaliação da QV relacionada ao traumatismo dentário ainda deve ser muito explorado a fim de poder de gerar subsídios para facilitar o desenvolvimento de estratégias de promoção de saúde para contemplar não somente o tratamento clínico do traumatismo dentário, mas, com uma visão holística do paciente, programar tratamento abrangendo também o âmbito psicossocial. 


\section{Colaboradores}

LAA Antunes participou na concepção e na redação final do artigo. AT Leão e LC Maia participaram na concepção, orientação e revisão crítica do trabalho.

\section{Agradecimentos}

Os autores agradecem o apoio do DAB/SAS/MS e DECIT/SCTIE/MS - CNPq e da FAPERJ.

\section{Referências}

1. Petersen PE, Bourgeois D, Ogawa H, EstupinanDay S, Ndiaye C. The global burden of oral diseases and risks to oral health. Bull World Health Organ 2005; 83(9):661-669.

2. Cortes MI, Marcenes W, Sheiham A. Impact of traumatic injuries to the permanent teeth on the oral health-related quality of life in 12-14-year-old children. Community Dent Oral Epidemiol 2002; 30(3):193-198.

3. Baratieri LN. Odontologia restauradora. Fundamentos e possibilidades. 1a Edição. São Paulo: Ed. Santos; 2002.

4. McGrath C, Broder H, Wilson-Genderson M. Assesing the impact of oral health on the life quality of children: implications for research and practice. Community Dent Oral Epidemiol 2004; 32(2):81-85.

5. Pal DK. Quality of life assessment in children: a review of conceptual and methodological issues in multidimensional health status measures. J Epidemiol Community Health 1996; 50(4):391-396.

6. Kuczynski E, Assumpção Jr FP. Definições atuais sobre o conceito de qualidade de vida na infância e adolescência. Pediatr Mod 1999; 35(3):73-78.

7. Ferreira RA. Odontologia: essencial para a qualidade de vida. Rev Assoc Paul Cir Dent 1997; 51(6): 514-521.

8. Jokovic A, Locker D, Stephens M, Kenny D, Tompson B, Guyatt G. Validity and reliability of a questionnaire of measuring child oralhealth- related quality of life. J Dent Res 2002; 81(7):459-463.

9. Locker D, Jokovic A, Stephens M, Kenny D, Tompson B, Guyatt G. Family impact of child oral and oro-facial conditions. Community Dent Oral Epidemiol 2002; 30(6):438-448.

10. Jokovic A, Locker D, Tompson B, Guyatt G. Questionnaire for measuring oral health-related quality of life in eight-to-ten-year-old children. Pediatr Dent 2004; 26(6):512-518.

11. Gherunpong S, Tsakos G, Sheiham A. Developing and evaluating an oral health-related quality of life index for children: the CHILD-OIDP. Community Dent Health 2004; 21(2):161-169.

12. Pahel BT, Rozier RG, Slade GD. Parental perceptions of children's oral health: The Early Childhood Oral Health Impact Scale (ECOHIS). Health Qual Life Outcomes 2007; 30(5):6.

13. Broder HL, McGrath C, Cisneros GJ. Questionnaire development: face validity and item impact testing of the Child Oral Health Impact Profile. Community Dent Oral Epidemiol 2007; 35(Supl. 1):8-19.

14. Goursand D, Paiva SM, Zarzar PM, Ramos-Jorge ML, Cornacchia GM, Pordeus IA, Allison PJ. Crosscultural adaptation of the Child Perceptions Questionnaire 11-14 (CPQ $\left.{ }^{11-14}\right)$ for the Brazilian Portuguese language. Health Qual Life Outcomes 2008; 14(6):2.

15. Barbosa TS, Tureli MC, Gavião MB. Validity and reliability of the Child Perceptions Questionnaires applied in Brazilian children. BMC Oral Health 2009; 18(9):13. 
16. Torres CS, Paiva SM, Vale MP, Pordeus IA, RamosJorge ML, Oliveira AC, Allison PJ. Psychometric properties of the Brazilian version of the Child Perceptions Questionnaire $\left(\mathrm{CPQ}_{11-14}\right)$ - short forms. Health Qual Life Outcomes 2009; 17(7):43.

17. Martins MT, Ferreira FM, Oliveira AC, Paiva SM, Vale MP, Allison PJ, Pordeus IA. Preliminary validation of the Brazilian version of the Child Perceptions Questionnaire 8-10. Eur J Paediatr Dent 2009; 10(3):135-140.

18. Goursand D, Paiva SM, Zarzar PM, Pordeus IA Grochowski R, Allison PJ. Measuring parental-caregiver perceptions of child oral health-related quality of life: psychometric properties of the Brazilian version of the P-CPQ. Braz Dent J 2009; 20(2):169-174.

19. Barbosa TS, Steiner-Oliveira C, Gavião MBD. Tradução e Adaptação Brasileira do Parental-Caregiver Perceptions Questionnaire (P-CPQ). Saúde Soc São Paulo 2010; 19(3):698-708.

20. Castro RA, Cortes MI, Leão AT, Portela MC, Souza IP, Tsakos G, Marcenes W, Sheiham A. Child-OIDP index in Brazil: cross-cultural adaptation and validation. Health Qual Life Outcomes 2008; 15(6):68.

21. Scarpelli AC, Oliveira BH, Tesch FC, Leão AT, Pordeus IA, Paiva SM. Psychometric properties of the Brazilian version of the Early Childhood Oral Health Impact Scale (B-ECOHIS). BMC Oral Health 2011; 13(11):19.

22. Barbosa TS, Gavião MBD. Evaluation of the Family Impact Scale for Use in Brazil. J Appl Oral Sci 2009; 17(5):397-403.

23. Goursand D, Paiva SM, Zarzar PM, Pordeus IA Allison PJ. Family Impact Scale (FIS): psychometric properties of the Brazilian Portuguese language version. Eur J Paediatr Dent 2009; 10(3):141-146.

24. Ramos-Jorge ML, Bosco VL, Peres MA, Nunes AC. The impact of treatment of dental trauma on the quality of life of adolescents - a case-control study in southern Brazil. Dent Traumatol 2007; 23(2):114-119.

25. Fakhruddin KS, Lawrence HP, Kenny DJ, Locker D. Impact of treated and untreated dental injuries on the quality of life of Ontario school children. Dent Traumatol 2008; 24(3):309-313.

26. Locker D. Disparities in oral health-related quality of life in a population of Canadian children. Community Dent Oral Epidemiol 2007; 35(5):348-356.

27. Locker D. Validity of single-item parental ratings of child oral health. Int J Paediatr Dent 2008; 18(6):407 414.

28. Bernabé E, Krisdapong S, Sheiham A, Tsakos G. Comparison of the discriminative ability of the generic and condition-specific forms of the ChildOIDP index: a study on children with different types of normative dental treatment needs. Community Dent Oral Epidemiol 2009; 37(2):155-162.

29. Berger TD, Kenny DJ, Casas MJ, Barrett EJ, Lawrence HP. Effects of severe dentoalveolar trauma on the quality-of-life of children and parents. Dent Traumatol 2009; 25(5):462-469.

30. Bendo CB, Paiva SM, Torres CS, Oliveira AC, Goursand D, Pordeus IA, Vale MP. Association between treated/untreated traumatic dental injuries and impacto $\mathrm{n}$ quality of life of Brazilian schoolchildren. Health Qual Life Outcomes 2010; 4(8):114.
31. Abanto J, Carvalho TS, Mendes FM, Wanderley MT, Bönecker M, Raggio DP. Impact of oral diseases and disorders on oral health-related quality of life of preschool children. Community Dent Oral Epidemiol 2011; 39(2):105-114.

32. Locker D. Measuring oral health: a conceptual framework. Community Dent Health 1988; 5(1):3-18.

33. Porrit JM, Rodd HD, Ruth Baker S. Quality of life impacts following childhood dento-alveolar trauma. Dent Traumatol 2011; 27(1):2-9.

34. Cardoso M, de Carvalho Rocha MJ. Traumatized primary teeth in children assisted at the Federal University of Santa Catarina, Brazil. Dent Traumatol 2002; 18(3):129-133.

35. Slade GD, Strauss RP, Atchison KA, Kressin NR, Locker D, Reisine ST. Conference summary: assessing oral health outcomes-measuring health status and quality of life. Community Dent Health 1998; 15(1):3-7.

36. Cardoso M, de Carvalho Rocha MJ. Traumatized primary teeth in children assisted at the Federal University of Santa Catarina, Brazil. Dent Traumatol 2002; 18(3):129-133

37. Granville-Garcia AF, de Menezes VA, de Lira PIC. Dental trauma and associated factors in Brazilian preschoolers. Dent Traumatol 2006; 22(6):318-322.

38. Oliveira LB, Marcenes W, Ardenghi TM, Sheiham A, Bönecker M. Traumatic dental injuries and associated factors among Brazilian preschool children. Dent Traumatol 2007; 23(2):76-81.

39. Ferreira JM, Fernandes de Andrade EM, Katz CR, Rosenblatt A. Prevalence of dental trauma in deciduous teeth of Brazilian children. Dent Traumatol 2009; 25(2):219-223.

40. Robson F, Ramos-Jorge ML, Bendo CB, Vale MP, Paiva SM, Pordeus IA. Prevalence and determining factors of traumatic injuries to primary teeth in preschool children. Dent Traumatol 2009; 25(2):118-122.

41. Soriano EP, Caldas Junior AF, Diniz de Carvalho MD, Amorim Filho HA. Prevalence and risk factors related to traumatic dental injuries in Brazilian schoolchildren. Dent Traumatol 2007; 23(4):232-240.

42. Traebert J, Bittencourt DD, Peres KG, Peres MA, Lacerda JT, Marcenes W. Aetiology and rates of treatment of traumatic dental injuries among 12year-old school children in a town in southern Brazil. Dent Traumatol 2006; 22(4):173-178.

43. Andreasen JO, Lauridsen E, Daugaard-Jensen J. Dental traumatology: an orphan in pediatric dentistry? Pediatr Dent 2009; 31(2):153-156.

44. Jesus MA, Antunes LA, Risso P de A, Freire MV, Maia LC. Epidemiologic survey of traumatic dental injuries in children seen at the Federal University of Rio de Janeiro, Brazil. Braz Oral Res 2010; 24(1): 89-94.

Artigo apresentado em 02/08/2011

Aprovado em 24/08/2011

Versão final apresentada em 13/10/2011 